\title{
ISLAM, DEMOCRACIA Y NEOLIBERALISMO: LOS HERMANOS MUSULMANES EGIPCIOS EN EL PODER
}

\author{
MARIELA CUADRO \\ Universidad de La Plata
}

\section{Introducción}

A partir de la preeminencia que adoptó el islam como identidad política en Medio Oriente, luego de la caída en desgracia del nacionalismo árabe, las baterías del orientalismo ${ }^{1}$ se han dirigido contra él. Así, los movimientos políticos islámicos fueron homogeneizados y construidos como "amenazas"; se hizo caso omiso de sus distintas experiencias y sus diversas coyunturas histórico-políticas de emergencia. Esto se debió a que tendió a primar la lectura religiosa, aquella que para explicar estos movimientos recurrió a los textos sagrados. ${ }^{2}$

Los levantamientos que tuvieron lugar en muchos países árabes a partir de 2010 destacaron la relevancia que tienen estos movimientos para las poblaciones, quienes allí donde existieron procesos electorales los acompañaron con sus votos, para colocarlos en importantes posiciones de poder; de este modo, muchos de ellos, al participar del juego democrático, pasaron de ser perseguidos y censurados a perfilarse como actores clave de la política regional. Esto fue percibido como amenaza por algunas monarquías del Golfo, que temieron ver socavada su base de poder nacional y regional; comenzaron entonces una campaña con el fin de desarticularlos, debilitarlos y desplazarlos de las nuevas posiciones adquiridas. La campaña supuso repre-

Este artículo fue recibido por la dirección de la revista el 1 de septiembre de 2015 y aceptado para su publicación el 25 de octubre de 2015.

${ }^{1}$ Edward Said, Orientalismo, Madrid, Libertarias, 1991.

${ }^{2}$ Véase, entre otros, Bernard Lewis, La crisis del islam. Guerra Santa y terrorismo, Barcelona, Ediciones B, 2003. 
sión, pero también construcción de consenso a través de una producción discursiva basada tanto en concepciones orientalistas como en la particular lectura acerca de la llamada Primavera Árabe que prevalecía.

Con el discurso mayoritario, la Primavera Árabe fue construida como un grito de libertad de las dormidas poblaciones árabes que, finalmente, se sumaban al tren de la historia. Un tren que, se sostenía, había partido desde Estados Unidos en el siglo XVIII, y vencido primero a los totalitarismos europeos y japonés, para pasar luego a hacer lo propio con las dictaduras de América Latina, y al fin arribar como última estación a las ex repúblicas soviéticas del este y del centro de Europa. Esta lectura, que homologaba libertad con democracia, echaba por tierra la tesis huntingtoniana de la imposibilidad cultural de los árabes-musulmanes de vivir la experiencia democrática.

Dos factores quedaban fuera de esta construcción discursiva: el económico y el religioso. En cuanto al primero, la crisis de las políticas neoliberales establecidas tras el fin de los nacionalismos panárabes, producto de programas de reestructuración y su consecuente profundización de desigualdades, estuvo ausente en la gran mayoría de los análisis que acompañaron los levantamientos; no obstante, si bien en estas lecturas la variable económica no ocupó un lugar como condición de posibilidad o causa de las revueltas, sí se sostuvo en varios espacios de poder de la política internacional que la reforma política (libertad igual a democracia) debía estar acompañada por una reforma económica que profundizara la libertad de mercado. ${ }^{3}$ En cuanto al factor religioso, por un lado, su falta fue remarcada (y festejada); por otro, ciertas lecturas subrayaron la posibilidad amenazante de una experiencia similar a la Revolución Islámica de Irán, de $1979 .{ }^{4}$

${ }^{3}$ En palabras del presidente de Estados Unidos, Barack Obama: "El objetivo debe ser un modelo en el cual el proteccionismo dé lugar a la apertura, en el que el reinado sobre el comercio pase de los pocos a los muchos, y la economía genere empleos para los jóvenes. El apoyo de Estados Unidos a la democracia estará basado, por lo tanto, en asegurar la estabilidad financiera, al promover la reforma e integrar los mercados competitivos entre sí y con la economía global" (The White House, "Remarks by the President on the Middle East and North Africa”, Washington, 19 de mayo de 2011).

${ }^{4}$ Para un mayor desarrollo de este punto, véase Mariela Cuadro, "Historia del pre- 
El artículo que aquí se presenta pretende abordar estas cuestiones a través de una mirada crítica que busca desesencializar los movimientos islámicos por medio del análisis específico del caso de los Hermanos Musulmanes en Egipto, al enfocarse en su política económica. Al respecto, algunos autores identifican los movimientos islámicos con las políticas neoliberales, ${ }^{5}$ mientras que otros plantean que ciertos principios religiosos conducen a estos movimientos a inclinarse por políticas económicas que subrayan la importancia de la justicia social y de la economía moral. ${ }^{6} \mathrm{El}$ presente trabajo postula que no existe una única práctica islámica económica, pues aparece condicionada por el contexto sociohistórico mundial y local en el que se despliega.

La elección de la unidad de análisis se explica tanto por el carácter particular que reviste la organización de los Hermanos Musulmanes egipcia, como por la importancia geopolítica de Egipto en la región; efectivamente, los Hermanos Musulmanes conforman el movimiento político islámico de mayor trayectoria en Medio Oriente y el Norte de África, con la especificidad de que se han expandido en la región y han aceptado las reglas del juego democrático. Por otra parte, con la mayor población árabe y una posición estratégica entre el Magreb y el Mashrek, y al ser el primer Estado árabe en haber reconocido al Estado de Israel, Egipto es de fundamental importancia en las relaciones de poder regionales. Los acontecimientos en Egipto afectan a la totalidad de Medio Oriente y el Norte de África.

sente: las revueltas árabes a través del prisma de las Revoluciones europeas de 1989-1990 y de la Revolución Islámica de Irán”, Revista de Relaciones Internacionales, vol. 20, núm. 40, 2011, pp. 131-159.

${ }^{5}$ Por ejemplo, Olivier Roy, "The Transformation of the Arab World", Journal of Democracy, vol. 23, núm. 3, 2012, pp. 5-18, y Charles Tripp, Islam and the Moral Economy. The Challenge of Capitalism, Cambridge, Cambridge University Press, 2006.

${ }^{6}$ Larbi Sadiki, Rethinking Arab Democratization: Elections without Democracy, Nueva York, Oxford University Press, 2009; Alberto Sánchez González y Carlos Calderón Modrego, "Banca islámica: un modelo de financiamiento alternativo para el desarrollo de África”, Relaciones Internacionales, núm. 26, junio-septiembre de 2014, pp. 69-84. 


\section{Los movimientos políticos islámicos, la democracia liberal y el neoliberalismo}

Aquí se trabajará sobre una forma específica adoptada por el islam político: aquella que acepta el juego democrático.

El significante democracia reviste especial interés en la política internacional contemporánea, ya que ha hegemonizado buena parte de su discurso. Como se planteó en la Introducción, aparece en el discurso internacional homologado con el concepto de libertad. Y como este último concepto fue situado por Michel Foucault en el centro del modo de ejercicio del poder liberal, ${ }^{7}$ al seguir al filósofo francés resulta posible llenar de contenido el significante democracia con un significado bien particular: el de democracia liberal, ${ }^{8}$ término que Chantal Mouffe definió como paradójico. ${ }^{9}$ Además de implicar un proceso técnico para la elección de los gobernantes, la democracia liberal es entendida como una relación social que supone dos elementos también fundamentales: un sujeto de gobierno construido como individuo-ciudadano, y un modelo económico neoliberal basado en la libertad de mercado, con la competencia como su principio organizador. Los dos últimos tienen incidencia en la relación de la democracia liberal con los movimientos políticos islámicos.

El debate acerca de la compatibilidad entre islam y democracia suele girar en torno de lecturas culturalistas que invocan la falta de antecedentes democráticos en los principales textos islámicos, ${ }^{10}$ o de la discusión sobre la posibilidad de separación entre religión y política en el islam. ${ }^{11} \mathrm{La}$ cuestión de los modos de subjetivación y su historización aporta una tercera

${ }^{7}$ Michel Foucault, Nacimiento de la biopolítica, Buenos Aires, FCE, 2007.

${ }^{8}$ Ido Oren, “The Subjectivity of the 'Democratic' Peace: Changing U.S. Perceptions of Imperial Germany”, International Security, vol. 20, núm. 2, 1995, pp. 147-184.

${ }^{9}$ Chantal Mouffe, "Carl Schmitt y la paradoja de la democracia liberal”, Tópicos, núm. 10, 2002, pp. 5-25.

${ }^{10}$ Véase, por ejemplo: Emmanuel Sivan, El islam radical. Teología medieval, política moderna, Barcelona, Bellaterra, 1997, y Samuel P. Huntington, El choque de civilizaciones y la reconfiguración del orden mundial, Buenos Aires, Paidós, 1997.

${ }^{11}$ Tal como se afirma en Nader Hashemi, Islam, Secularism and Liberal Democracy. Toward a Democratic Theory for Muslim Societies, Nueva York, Oxford University Press, 2009. 
variable que cabe tener en cuenta. Así, se entiende al individuociudadano como sujeto de gobierno propio de una racionalidad de poder liberal, que existe junto a otros modos de subjetivación política, como el de súbdito o el de ciudadano de la polis griega, que corresponden a otras racionalidades de poder. En esta construcción, el proceso de secularización atravesado por Europa desempeñó un importante rol, pues implicó la creación de los ámbitos público y privado, y restringió a este último a la religión, la cual, así, fue desvinculada del espacio político; de esta manera, paulatinamente, la identidad religiosa que constituía a los europeos fue dando paso a nuevas identidades sociales ligadas a la emergencia de los Estados-nación y la idea de ciudadanía. Aún más: la religión cambió de significado. De acuerdo con Scott Thomas ${ }^{12}$ dejó de ser entendida en un sentido social, como referencia a una comunidad que constituye al sujeto, y pasó a ser considerada como un conjunto de doctrinas al que los individuos se adscriben voluntariamente. Esta última concepción, propia de la subjetividad liberal, choca con la concepción de los movimientos políticos islámicos no secularizados, que conciben al sujeto como constituido por la religión y no a la inversa.

La dimensión económica de la democracia liberal, como se afirmó anteriormente, tiene su eje en el libre mercado organizado por la competencia. Tal como postula Foucault, la diferencia entre esta última y el intercambio radica en que la libre competencia se sostiene sobre la desigualdad. Si para la racionalidad neoliberal es el libre mercado el regulador no sólo de la economía sino también de la sociedad en su conjunto, la desigualdad - aquello que permite, por ejemplo, el funcionamiento del mecanismo regulador de precios- también organiza la sociedad. En palabras del filósofo francés: para el neoliberalismo

[...] la relativa igualación no puede en ningún caso constituir un objetivo. No puede constituir un objetivo en un sistema en el cual, justamente, la regulación económica, es decir, el mecanismo de los precios, no se obtiene en absoluto a través de los fenómenos de igualación sino por un

${ }^{12}$ Scott Thomas, The Global Resurgence of Religion and the Transformation of International Relations. The Struggle for the Soul of the Twenty-first Century, Nueva York, Palgrave Macmillan, 2005. 
juego de diferenciaciones que es característico de cualquier mecanismo de competencia y se establece a través de oscilaciones que sólo cumplen su función y sus efectos reguladores siempre que, desde luego, se las deje actuar, y mediante las diferencias. En términos generales, es preciso que haya algunos que trabajen, y otros que no trabajen o bien que haya salarios grandes y pequeños, que los precios suban y bajen, para que las regulaciones actúen..$^{13}$

Ahora bien, para la emergencia del neoliberalismo, entendido como racionalidad que aspira al gobierno de la economía sobre la sociedad, tuvo que existir previamente un proceso por el cual, en primer lugar, pudieran pensarse ambas esferas -la económica y la social- como separadas. Esto conduce nuevamente al proceso de secularización. Efectivamente, el proceso de secularización en el campo de la subjetividad fue concomitante con aquel que tuvo lugar en el campo del conocimiento y que dio lugar a la ciencia y, dentro de ella, las disciplinas. Las tres más relevantes en las ciencias sociales fueron la economía, la ciencia política y la sociología; las tres respondían a la racionalidad liberal que definía la modernidad a partir de la diferenciación de tres esferas sociales: el mercado, el Estado y la sociedad civil. "Las tres esferas operaban, se sostenía, de acuerdo con diferentes lógicas, y era bueno mantenerlas separadas una de otra". ${ }^{14}$ De esta manera, no sólo la esfera política se desvinculó de la religiosa, sino que también la económica fue separada de la política; por lo tanto, también religión y economía comienzan a ser supuestos como dos ámbitos desvinculados.

Ahora bien, según Nader Hashemi, una de las características de los movimientos políticos islámicos es, precisamente, que no conciben la existencia de estas esferas como separadas. ${ }^{15}$ Olivier Roy refrenda la última afirmación, y sostiene que el concepto de economía islámica es una creación del siglo xx, ya que "en la tradición islámica temprana, la economía no era considerada un agente autónomo". ${ }^{16}$ Esto permite comprender

\footnotetext{
${ }^{13}$ Foucault, Nacimiento de la biopolítica, op. cit., p. 176. Itálicas agregadas.

${ }^{14}$ Immanuel Wallerstein, World-Systems Analysis. An Introduction, DurhamLondres, Duke University Press, 2004, p. 6.

${ }^{15}$ Hashemi, Islam, Secularism and Liberal Democracy, op. cit.

${ }^{16}$ Olivier Roy, The Failure of Political Islam, Cambridge, Harvard University Press, 1994.
} 
que uno de los rasgos de la economía islámica sea su carácter moral, si se entiende la economía moral como una integrada a la sociedad en su conjunto, es decir, una economía cuyos objetivos, finalidades y procesos están dirigidos y conformados por instituciones extraeconómicas. ${ }^{17}$

Larbi Sadiki sostiene y desarrolla la noción de que la economía moral tiene fuertes bases en las sociedades predominantemente islámicas. En primer lugar, plantea la vinculación entre la economía islámica y los mandatos coránicos contra el consumo excesivo, la usura y el robo. Asevera que "la riqueza de un musulmán está gobernada por obligaciones comunales que subrayan el derecho (baqq) de los necesitados-pobres (al-miskin), los huérfanos (al-yatim) y los caminantes (ibn alsabil)". ${ }^{18}$ Para el autor, el fin de la actividad económica es asegurar que los musulmanes sean provistos de un ambiente que facilite la práctica del islam y maximice el sometimiento a Dios y no las distracciones pecuniarias o las preocupaciones por la sobrevivencia. Sadiki concluye que el corolario de esta economía moral es la primacía de la justicia social dentro del marco islámico: "Las formulaciones de la justicia social en el islam pueden variar en la práctica y en su alcance de acuerdo con el contexto, pero lo que hace su concepción paradigmáticamente distintiva y común a todas las sociedades musulmanas es su sistema redistributivo basado en la comunidad". ${ }^{19}$ Según el autor, esto explica que el bienestar social sea una prioridad para los movimientos islámicos.

En este sentido, aunque limitados, es válido repasar algunos elementos económicos del islam. En principio hay que aclarar que el islam no se opone a la propiedad privada; sin embargo, sí hace hincapié en la obligatoriedad de la distribución de la riqueza: le está permitido a un musulmán acumular dinero, siempre y cuando una parte de él sea colocado al servicio de la comunidad (umma) a través de instituciones de caridad. Éstas han sido históricamente las que han provisto a la sociedad de

${ }^{17}$ Basak Ozaral, "Islam and Moral Economy”, en Tugrul Keskin (ed.), The Sociology of Islam. Secularism, Economy and Politics, Londres, Ithaca Press, 2011, pp. 21-44.

${ }^{18}$ Sadiki, Rethinking Arab Democratization, op. cit., p. 202.

${ }^{19}$ Ibid., p. 203. 
servicios de salud y educación. ${ }^{20}$ Así, como establece Sergio Moya Mena, "el valor de la igualdad tiene en el islam un peso más importante que la idea de libertad [...] El islam tiene un fuerte sentido igualitario". ${ }^{21}$

Una de las principales instituciones islámicas que refiere a la cuestión económica es el waqf, una donación religiosa inalienable de propiedades en beneficio de la comunidad, a la que los musulmanes son alentados. Además, el zakat, la prohibición de riba y de gharar son los tres principales instrumentos de la economía moral islámica. El primero adopta la forma de un impuesto compulsivo para la caridad, lo que lo transforma en un elemento fundamental para políticas de bienestar y distribución equitativa de la riqueza. Equivale a $2.5 \%$ anual de la riqueza total del sujeto obligado. Ahmed El-Ashker y Rodney Wilson establecen una vinculación entre el zakat y la seguridad social provista por el Estado, al plantear que, según lo teorizado por Al-Fangari, la última implica las obligaciones del Estado hacia sus ciudadanos para proveer la ayuda necesaria a los pobres y los ancianos. Según el mismo autor, esta obligación aparece enunciada tanto en el Corán como en la Sunna. ${ }^{22}$

En cuanto al riba, es la prohibición de cobrar interés, bajo el supuesto de que el islam se opone a la explotación. En este marco, sostienen sus defensores, cobrar interés a alguien que se ha visto obligado a pedir un préstamo es una forma de explotación económica. "El riba es considerado una forma de opresión porque, se argumenta, transfiere riqueza de los pobres hacia los ricos y contribuye a la desigualdad en la distribución de la riqueza en la sociedad". ${ }^{23}$ Debido a las dificultades que plantea el sistema financiero capitalista relativo a esta prohibición, los bancos islámicos han proliferado. Finalmente, la economía islámica prohíbe también el gharar, es decir, situaciones de falta de claridad en el marco de transacciones económicas.

${ }^{20}$ Una de las razones que explica el triunfo de los Hermanos Musulmanes en Egipto es que ante la retirada del Estado de los ámbitos sociales, debido a las reformas neoliberales, la agrupación islámica pasó a llenar el vacío dejado por él.

${ }^{21}$ Sergio Moya Mena, "El islam y sus manifestaciones sociopolíticas contemporáneas: breve introducción" (inédito). Itálicas agregadas.

${ }^{22}$ Ahmed El-Ashker y Rodney Wilson, Islamic Economics: A Short History, Boston, Brill, 2005.

${ }^{23}$ Ozaral, "Islam and Moral Economy", op. cit., p. 37. 
Más allá de estos elementos en común que, podría afirmarse, tienden hacia la justicia social y la igualdad, los modos que adopta la economía en los distintos contextos históricos, internacionales y nacionales en los que el islam político se despliega explican la diversidad de miradas sobre la relación entre éste y el neoliberalismo. Por ejemplo, Charles Tripp afirma que la posibilidad de conjugación entre los preceptos de la justicia social islámica y el sistema económico capitalista llevó, durante la década de 1990, a la justificación de una economía islámica neoliberal que subrayaba la idea de libre competencia y la prohibición del monopolio como puntos nodales. ${ }^{24}$ En la misma línea, Roy plantea que, al ser "amigos del statu quo cuando se trata de asuntos económicos", ${ }^{25}$ los islamistas se contentan con dirigir instituciones caritativas en barrios pobres, pero se oponen a las huelgas y las reformas agrarias. No obstante, es válido aclarar que, en un trabajo anterior, el académico francés postula la existencia de por lo menos dos modelos económicos islámicos: uno graficado por los Hermanos Musulmanes y el Irán posrevolucionario, tendente a la justicia social; y otro condensado por el Frente Islámico de Salvación argelino y el Partido de la Prosperidad turco, que se encuentra fuertemente influido por los preceptos neoliberales. ${ }^{26}$

\section{Los Hermanos Musulmanes en contexto: el neoliberalismo en Egipto}

Egipto fue parte de la ola neoliberal que a partir de la década de 1970 inundó el mundo. Luego del fin del gobierno de Gamal Abdel Nasser, la política de su sucesor, Anwar Al-Sadat, estuvo dirigida a lograr un acercamiento con Estados Unidos en detrimento de la relación que, hasta el momento, El Cairo había tenido con la Unión Soviética.

La infitab es en parte explicada por este giro hacia Occidente. Se trató de un proyecto de liberalización económica y política que no llegó a concretarse durante la presidencia de quien

\footnotetext{
${ }^{24}$ Tripp, Islam and the Moral Economy, op. cit.

${ }^{25}$ Roy, "The Transformation of the Arab World", op. cit., p. 13.

${ }^{26}$ Roy, The Failure of Political Islam, op. cit.
} 
fuera asesinado en 1981. Karatholuvu Nagarajan señala que la infitah puede ser explicada a través de una ecuación:

$$
\begin{gathered}
\text { Capital árabe }{ }^{27}+\text { tecnología occidental } \\
+ \text { recursos egipcios = desarrollo y progreso. }{ }^{28}
\end{gathered}
$$

"El argumento subyacente era el clásico argumento económico estándar: permitan a las fuerzas del mercado operar libremente y los recursos serán asignados eficientemente, lo que abrirá paso a la prosperidad económica. El principio de equidad que figuró de manera prominente durante la temprana era de Nasser fue minimizado, y aun completamente ignorado". ${ }^{29} \mathrm{El}$ intento de recortes de subsidios, establecidos durante el gobierno nacionalista, a pedido del Fondo Monetario Internacional (FMI), en 1976, a cambio de un préstamo para subsanar la balanza de pagos egipcia, y los consecuentes levantamientos populares de 1977 que los impidieron, dan cuenta de un proyecto político a favor de la libertad de mercado y en detrimento de políticas de justicia social.

Hosni Mubarak, presidente derrocado durante los levantamientos de 2011, fue el encargado de llevar adelante el programa neoliberal. El préstamo otorgado por el FMI al gobierno egipcio, en 1991, puede ser considerado como el hito que marca el aterrizaje del neoliberalismo en el país árabe. A cambio de éste, el organismo financiero internacional pidió a El Cairo el establecimiento de un programa de reforma estructural de su política económica; esto implicaba, entre otras cosas, la disminución del déficit fiscal. Con tal fin, durante este periodo, $\mathrm{El}$ Cairo privatizó varias empresas públicas (entre 1991 y 1999 el Estado egipcio vendió propiedades por 4172 millones de dólares), ${ }^{30}$ estableció nuevas leyes de inversión y flexibilizó

${ }^{27}$ Capital de los países del Golfo, principalmente Arabia Saudita, que habían acumulado ingentes sumas de dinero con el recorte de la producción y el embargo petrolero de 1973.

${ }^{28}$ Karatholuvu Nagarajan, "Egypt's Political Economy and the Downfall of the Mubarak Regime", International Journal of Humanities and Social Science, vol. 3, núm. 10, 2013, pp. 22-39.

${ }^{29}$ Ibid., p. 27.

${ }^{30}$ Adam Hanieh, Lineages of Revolt. Issues of Contemporary Capitalism in the Middle East, Chicago, Haymarket, 2013. 
los controles burocráticos. Esta tendencia se acentuó a partir de 1995, año de ingreso de Egipto a la Organización Mundial de Comercio.

El resultado fue el aumento de la desigualdad, bajo la forma del crecimiento del desempleo y la pobreza. Respecto al sector rural, fueron interrumpidos los subsidios agrícolas y desmanteladas las cooperativas establecidas durante los años nasseristas. Asimismo, el derecho a la tenencia de la tierra que había beneficiado a los campesinos durante el gobierno de Nasser fue revocado en 1992, y las relaciones entre los propietarios de las tierras y los arrendatarios cambiaron en favor de los primeros. El sector laboral, desregulado, también fue afectado, al declinar el Estado su prerrogativa de determinar salarios mínimos y supervisar la resolución de conflictos entre empresas y trabajadores.

Las privatizaciones fundaron una nueva clase social formada por quienes se beneficiaron de ellas. A partir de 2000, con el ascenso político meteórico del hijo del ex presidente, Gamal Mubarak, esta clase de empresarios comenzó a ocupar cada vez más espacios en el gobierno egipcio, lo que profundizó el proyecto neoliberal. El nombramiento, en 2004, de Ahmed Nazif como primer ministro aceleró el proceso, colocó a muchos representantes de este sector en puestos ministeriales y aumentó las ventas de compañías estatales de los sectores energético, petroquímico y financiero. Los compradores eran capitales extranjeros, capitales del Golfo o conglomerados de capitales nacionales, asociados, en general, con corporaciones extranjeras.

En 1974, cuando la infitab fue anunciada, los Hermanos Musulmanes se opusieron, pues sostenían, por un lado, que una apertura hacia Occidente implicaría la erosión de los valores islámicos y, por otro, que beneficiaría sólo a unos pocos miembros de las capas más altas de la sociedad. En respuesta a la retirada del Estado, los Hermanos Musulmanes instalaron una red de servicios sociales que más tarde les habilitaría la victoria en las únicas elecciones libres de la historia de Egipto. 


\section{Los Hermanos Musulmanes en el juego democrático: su programa de gobierno}

Una gran cantidad de páginas se han escrito sobre el levantamiento que llevó a la caída de Mubarak en el marco de la crisis del modelo de acumulación arriba reseñado. Luego de un periodo transicional de gobierno del Consejo de Seguridad de las Fuerzas Armadas, a mediados de 2012, los Hermanos Musulmanes, bajo la rúbrica de Partido de la Libertad y la Justicia (PLJ), se impusieron en las primeras elecciones presidenciales democráticas de la historia de Egipto con su candidato Mohamed Morsi. Éste estuvo al frente del gobierno egipcio durante un año: el 3 de julio de 2013 fue derrocado por un golpe de Estado, cívico-militar, apoyado por algunas monarquías del Golfo, principalmente, Arabia Saudita, Emiratos Árabes Unidos y Kuwait.

A pesar de que muchos autores han planteado que los Hermanos Musulmanes no tenían un programa económico para gobernar Egipto en el contexto de una fuerte crisis, ${ }^{31}$ la organización ha defendido su programa económico. Al respecto, en agosto de 2012, en un comunicado difundido en su página web, el doctor Abdullah Khattab, miembro del Comité de Asuntos Económicos del PLJ, sostuvo que la visión económica de la organización planteaba un equilibrio "entre los sistemas de mercado del capitalismo y aquellos orientados al socialismo, dentro de un marco moral y la referencia islámica". ${ }^{32}$ De este modo, afirmó que los Hermanos Musulmanes, con base en el islam, intentaban un balance entre el libre mercado y los requerimientos de justicia social, pues buscaban construir instituciones fuertes y colocarlas fuera del control de las corporaciones empresarias:

En efecto, el compromiso de la Hermandad con un sistema económico con referencia islámica da a los negocios y a los hombres de negocios libertad para invertir, sin permitirles controlar la economía o los re-

${ }^{31}$ Véase, por ejemplo, Lorenzo Vidino, The Muslim Brotherhood after the Arab Spring: Tactics, Challenges and Future Scenarios, Bruselas, Wilfried Martens Centre for European Studies (Policy Brief), 2013.

${ }^{32}$ Ikhwanweb, "Khattab: Muslim Brotherhood Economic Program is based on Free Market, Social Justice”, El Cairo, 23 de agosto de 2012. 
cursos del Estado ni la dirección económica. Ciertamente, no permite la creación de un sistema de mercado completamente libre como aquel que dominaba la economía bajo el régimen anterior. ${ }^{33}$

El programa partía de un diagnóstico que identificaba como problemas económicos los siguientes: altos niveles de inflación e imposibilidad de los salarios de alcanzar las tasas inflacionarias; altos niveles de desempleo; altas tasas de pobreza y distribución de la riqueza inequitativa, con una proporción de pobres de cerca de $40 \%$ de la población; déficit de bienes estratégicos, especialmente trigo y algodón, y aumento de los precios en el ámbito global, especialmente de los bienes de consumo alimentarios, entre otros. ${ }^{34}$

El plan económico para abordar y solucionar estos problemas incluía los siguientes puntos: i) lograr la autosuficiencia en la producción de bienes estratégicos (especialmente, algodón y trigo); ii) alentar la participación activa de las pequeñas y medianas empresas en el desarrollo industrial egipcio; iii) activar el trabajo de las instituciones de caridad, alentar el cumplimiento con la obligación del zakat y reformar el sistema nacional de crédito caritativo, de modo tal que funcione para proveer a la comunidad; iv) revisar el programa de subsidios a las exportaciones; v) modificar los acuerdos de exportación de energía a fin de lograr precios de venta justos para beneficio de Egipto; ${ }^{35}$ vi) modificar y activar la ley de protección de la competencia, y evitar prácticas monopólicas a través del aumento de las penalidades, así como activar el rol de las autoridades de protección de la competencia, y vii) revisar el sistema de sueldos, establecer límites máximos y mínimos, multiplicar los sueldos constantes y disminuir los variables. ${ }^{36}$

Este plan económico - del cual se presenta sólo un resumen- estaba acompañado por un programa de justicia social,

${ }^{33}$ Idem.

${ }^{34}$ Ikhwanweb, “FJP’s 2011 Program: Security, Economy, and Corruption as Urgent Issues”, El Cairo, 4 de diciembre de 2011.

${ }^{35}$ Este punto estaba dirigido fundamentalmente a los acuerdos firmados con el Estado de Israel a partir de 2005, vinculados con una de las cláusulas de los Acuerdos de Paz de Camp David de 1979-1980. En abril de 2012, cuando los Hermanos Musulmanes lograron mayoría parlamentaria en el gobierno egipcio, el acuerdo de 2005 fue cancelado.

${ }^{36}$ Idem. 
pues el PLJ consideró que la "falta de justicia social" 37 había sido una de las principales causas de los levantamientos en Egipto; por lo tanto, el programa de gobierno presentado para la carrera presidencial postulaba la justicia social y la distribución de la riqueza como "algunas de las más importantes obligaciones del Estado". ${ }^{38}$ En este marco, de llegar a la presidencia, el partido se comprometía a tratar tres cuestiones fundamentales: el elevado costo de vida, la pobreza y el desempleo. Como medios para lograr este fin se proponía: la "recuperación de los fondos saqueados al Estado", ${ }^{39}$ la reforma del sistema impositivo, la promoción de los sistemas del zakat y el waqf, y el combate a la corrupción y al despilfarro de los recursos del Estado.

Las medidas para abordar el alto costo de vida estaban íntimamente vinculadas al programa económico, de tal modo que no se concebía la "esfera" económica separada de la social. Entre otros puntos, el PLJ destacaba los siguientes: la activación de la ley de protección de la competencia, de que no existieran prácticas monopólicas de fijación de precios; el mantenimiento de la prerrogativa del Estado sobre la fijación de precios y el control del mercado para productos básicos (este punto aclaraba que se trataba de medidas excepcionales para periodos limitados); la búsqueda de la autosuficiencia en los bienes básicos, como trigo, azúcar, petróleo, carne y algodón; la racionalización y el ajuste de la política de privatizaciones, y hacerla más clara, especialmente en lo concerniente a las industrias estratégicas, y la promoción de la producción local y la racionalización de las importaciones.

El punto relativo al programa de privatización mereció un desarrollo específico, pues se postulaba que se hacían necesarias nuevas formas de privatización, más efectivas y “apropiadas para la economía egipcia”. ${ }^{40}$ En este sentido, resultaba imperante evitar la privatización de "industrias estratégicas como la farmacológica, la alimentaria, la energética” y, en caso de que fuera necesario, se proponía que debían evaluarse las inversiones de de 2011.

${ }^{37}$ Ikhwanweb, “FJP 2011 Program on Social justice”, El Cairo, 4 de diciembre

${ }^{38}$ Idem.

${ }^{39}$ Idem.

${ }^{40}$ Idem. 
forma justa y "con total transparencia”, y dársele prioridad en la compra a los inversionistas nacionales.

Por su parte, la erradicación de la pobreza y la provisión de servicios sociales básicos aparecían vinculados al zakat y el waqf, definidos como uno de los modos que el "islam usa para combatir la pobreza, eliminar el desempleo, lograr la justicia social y económica, fortalecer la solidaridad social y la cohesión, y evitar la acumulación de la riqueza en las manos de unos pocos". ${ }^{41}$ Asimismo, para trabajar por la distribución de la riqueza de modo tal de "asegurar que cada uno tenga suficientes ingresos para cubrir los requerimientos mínimos de la vida", ${ }^{42}$ se planteaba la necesidad de aprobar una ley que impusiera salarios mínimos y máximos.

Finalmente, para lidiar con el desempleo se proponía, en primer lugar, crear empleo productivo de largo plazo, lo cual suponía aumentar en 30\% del producto interno bruto los niveles de inversión nacional. Respecto a la inversión extranjera directa, se planteaba su uso eficiente, y evitar su concentración en las industrias extractivas. Asimismo, se establecía la necesidad de poner un límite al desarrollo tecnológico en la industria, y alentar las tecnologías y las industrias intensivas en mano de obra. Por otra parte, el PLJ asumía enmendar la legislación relativa a los sindicatos, para darles mayor libertad y evitar su fragmentación, al tiempo que esperaba organizar el sindicato de trabajadores de la agricultura y la irrigación. Un apartado del programa, aún en análisis, está dedicado al problema de los chicos de la calle, sobre el cual propone una solución distinta a la vinculada con la seguridad.

Como formas para aumentar los recursos del Estado y así mejorar la situación presupuestaria, se proponía: cobrar el valor de la tierra regalada a empresarios e inversores, tales como las tierras de Madinati y el golfo de Suez; revisar los contratos de exportación de gas natural, "especialmente a Israel”; ${ }^{43}$ verificar los precios de exportación de materia prima y recursos minerales, "especialmente el petróleo exportado a Israel, que es exportado a un precio fijo de 6.8 dólares el barril desde la

${ }^{41}$ Idem.
${ }^{42}$ Idem.
${ }^{43}$ Idem. 
firma de los Acuerdos de Camp David"; ${ }^{44}$ eliminar los subsidios a las industrias de consumo intensivo de energía y revisar la política de subsidios a los productos derivados del petróleo "que da a los ricos $80 \%$ de los fondos"; ${ }^{45}$ verificar los precios de la electricidad para los segmentos de mayor consumo; cobrar los impuestos impagos de los sectores de mayores ingresos; hacer mejor uso de los préstamos del exterior y no recurrir a ellos a menos que existieran estudios de viabilidad y de preparación para su implementación.

Así, el programa presentaba una combinación de unas pocas políticas vinculadas con un modelo de acumulación neoliberal, y otras, mayoritarias, que apuntaban fuertemente al logro de la igualdad social. En este sentido, era representativo del equilibrio que desplegaron los Hermanos Musulmanes, al intentar llevar a cabo su plan de gobierno sin enemistar a las potencias liberales ni a las instituciones internacionales a ellas asociadas. De hacerse efectivo, implicaba afectar intereses creados muy arraigados en Egipto; la corporación contribuiría con $25 \%$ del producto interno bruto y el resto de la riqueza quedaría en manos de una burguesía no afecta a pagar sus impuestos. Autores como Maha Azzam llaman la atención sobre la coincidencia de que la intensificación de la oposición a Morsi en la calle haya tenido lugar poco después del discurso dado por el entonces presidente egipcio, en octubre, en el marco de sus cien días de mandato. Entonces afirmó que llevaría adelante el proyecto de su partido de perseguir a los sectores acaudalados con deudas impositivas. ${ }^{46}$

\section{Los Hermanos Musulmanes en el poder y los organismos financieros internacionales}

Quizá la lectura más representativa de lo que esperaban los sectores hegemónicos mundiales que resultara de los levantamientos árabes fue la del Grupo de los Ocho. En la Cumbre

${ }^{44}$ Idem.

${ }^{45} \mathrm{Idem}$.

${ }^{46}$ Maha Azzam, "Egypt's Democratic Experiment: Challenges to a Positive Trajectory”, Insight Turkey, vol. 15, núm. 2, 2013, pp. 157-169. 
de Deauville, de mayo de 2011, los Estados miembros consensuaron una declaración que definía los levantamientos árabes como "históricos" ${ }^{47}$ y portadores del "potencial para abrir la puerta al tipo de transformaciones ocurridas en Europa Central y Oriental luego de la caída del Muro de Berlín. La aspiración de la gente a la libertad, los derechos humanos, la democracia, las oportunidades laborales, el empoderamiento y la dignidad, la ha llevado a tomar el control de su propio destino". ${ }^{48}$ Lo cual, se afirmaba, "resuena con nuestros valores comunes y los refuerza". ${ }^{49}$ La Primavera Árabe era leída, así, como un grito "legítimo que pedía sociedades democráticas, abiertas, y modernización económica inclusiva". ${ }^{50}$

El tono fuertemente liberal de la Declaración es destacable. El Grupo de los Ocho se sumaba, de esta manera, a la lectura - mencionada en la Introducción- de que los levantamientos árabes formaban parte de un movimiento necesario de la historia que había tenido su último capítulo en la rebelión de los pueblos europeos centrales y orientales contra el yugo soviético. Por otra parte, además de la reforma política y social, se hacía hincapié en la necesidad de la reforma económica. Así, el capital financiero internacional y sus instituciones aprovecharon las protestas para instalar una agenda que apuntalara las políticas neoliberales ya establecidas por los gobiernos en jaque.

La Declaración hacía referencia al FMI, y daba la bienvenida a la construcción de marcos macroeconómicos en los países mencionados; asimismo, con el fin de mejorar el clima de negocios, los bancos multilaterales de desarrollo también eran llamados a la acción. En este sentido, se planteaba la necesidad de hacer uso de la experiencia del Banco Europeo para la Reconstrucción y el Desarrollo. Creado en 1990, este banco estuvo dedicado a dirigir la transición de las economías de las ex repúblicas soviéticas hacia economías neoliberales. La Declaración del Grupo de los Ocho llamaba a crear una extensión del Banco Europeo, "con el objetivo de apoyar la transición en los

${ }^{47}$ G8, "Declaration of the G8 on the Arab Spring", Cumbre de Deauville, Deauville, 26 y 27 de mayo de 2011.

${ }^{48} \mathrm{Idem}$.

${ }^{49}$ Idem.

${ }^{50}$ Idem. 
países de la región que abracen la democracia multipartidaria, el pluralismo y las economías de mercado". ${ }^{51}$

Por su parte, los países miembros prometían colaborar para mejorar las "oportunidades mutuas de acceso al mercado, con el fin de alentar la integración a la economía global a través del crecimiento del comercio y las inversiones en los países que están llevando a cabo reformas con el objetivo de abrir sus economías y crear condiciones competitivas"..$^{52}$ Con esta finalidad, eran destacadas las negociaciones encaminadas a lograr acuerdos de libre comercio. Respecto a Egipto, en particular, se daba la bienvenida a la decisión de las autoridades de pedir asistencia al FMI y bancos de crédito internacionales.

Por su parte, el FMI leyó en el "despertar árabe" una demanda de cambio no sólo político, sino también económico. En un discurso, pronunciado en la London School of Economics en noviembre de 2012, el primer subdirector gerente del Fondo, David Lipton, analiza positivamente el proceso de reformas llevado a cabo por los países árabes a partir de la década de 1980, aunque señala que continúa el problema de la generación de empleos.

Ante este diagnóstico, y con el objetivo de lograr un crecimiento sustentable, se recomendaba moverse de la inversión estatal a la privada, y de las industrias protegidas a un crecimiento dirigido hacia la exportación y la creación de valor. De esta manera, para "destrabar el crecimiento, de formidable potencial, el sector privado debe convertirse en su principal fuente, lo cual sólo va a suceder si puede acceder a los mercados globales, y no únicamente a los locales”. ${ }^{53}$ Tal era el significado de la "integración comercial", lo cual debía subrayarse. El principio ordenador era la competencia: "Un país que se abre a la competencia internacional encontrará, inevitablemente, mayor coherencia en el resto de las reformas, porque estas reformas le ayudarán a competir".${ }^{54} \mathrm{El}$ comercio era pensado, entonces, en términos de competencia integrada.

${ }^{51}$ Idem.

${ }^{52}$ Idem.

${ }^{53}$ David Lipton, "Enabling Economic Transformation in the Middle East and North Africa”, discurso en la London School of Economics, 13 de noviembre de 2012.

${ }^{54}$ Idem. 
El listado de recomendaciones incluía: mejorar el clima de negocios y, por lo tanto, disminuir las regulaciones; reformar el mercado de trabajo "para reducir los obstáculos a la contratación”; mejorar la educación y alinearla a los requerimientos de los empleadores privados, a fin de alentar las aptitudes técnicas; facilitar el acceso a las finanzas, mejorando y alentando al sector bancario; y reemplazar con una "red moderna de seguridad social" los subsidios no dirigidos. ${ }^{55}$

A pesar de que se manifestaba que la solución dependía de cada uno de los países implicados, se consideraba que la "comunidad internacional" tenía un rol que desempeñar. Así, se destacaba la ayuda prestada por los "socios de Medio Oriente y Norte de África”, Estados Unidos, la Unión Europea y los países del Consejo de Cooperación del Golfo. Lipton también hizo referencia, como modelo, a la acción del Banco Europeo para la Reconstrucción y el Desarrollo en Europa central y oriental, pues subrayó que las inversiones a través de dicha institución "no sólo catalizarán la transformación económica, sino que también servirán a los intereses económicos y de seguridad de la comunidad internacional”. ${ }^{56}$

En mayo de 2011, el gobierno de transición del Consejo de Seguridad de las Fuerzas Armadas pidió un préstamo al FMI, con el cual aspiraba a conseguir entre 10000 y 12000 millones de dólares. El organismo internacional aceptó la entrega de 3000 millones, pero, en junio, el gobierno lo rechazó, pues adujo que podía achicar el déficit fiscal sin necesidad de ese dinero. Es importante destacar que durante los levantamientos en Egipto existieron planteamientos en contra del FMI, al que se vinculaba con la política económica de exclusión del ex presidente Mubarak.

Un año después de la Declaración de Deauville, el Partido de la Libertad y la Justicia había asumido el poder político en Egipto. En agosto de 2012, a poco de asumir la presidencia, el partido vinculado a los Hermanos Musulmanes emitió un comunicado en el que planteaba algunas razones para negociar un préstamo con el FMI; entre ellas, destacaba su importancia no 
tanto para cubrir el déficit estatal heredado, que llegaba a los 29000 millones de dólares a fines de junio de $2012,{ }^{57}$ sino para lograr "la confianza de las potencias mundiales y las instituciones de la economía egipcia” ${ }^{58}$ Es decir, una negociación exitosa con el FMI - que tenía como condición, claridad y transparencia por parte de El Cairo- no tenía como objetivo resolver la crítica situación económica egipcia, sino, fundamentalmente, lograr confianza internacional. Por otra parte, no pasaba desapercibido el hecho de que un acuerdo con el FMI implicaría un programa de reformas que afectaría "las políticas futuras de la economía egipcia" ${ }^{59}$ razón por la cual se urgía a privilegiar los intereses de Egipto y de los ciudadanos de bajos ingresos, quienes, se señalaba, no debían ser perjudicados.

La cuestión de la generación de empleo -que, según el FMI, había aumentado de $9 \%$ a fines de 2010 a 12\% luego de los levantamientos- ocupó un lugar destacado en las recomendaciones formuladas por el organismo internacional. Con tal fin se recomendaba la creación de una economía "más transparente y competitiva”, y se hacía alusión al fuerte rol de la burocracia estatal egipcia en el control de la economía. Para lograr un crecimiento sostenible inclusivo se consideraba necesario un ambiente macroeconómico estable, para lo cual, a su vez, urgía la consolidación fiscal. Esta última era posible a través de la "modernización" del sistema impositivo mediante la incorporación de un impuesto al valor agregado (IVA). Es menester destacar que la preocupación por la generación de empleo no era nueva en el discurso del FMI: según Hanieh, también había formado parte de los planteamientos que precedieron a la instauración del neoliberalismo en Egipto. ${ }^{60}$ Respecto del IVA, varios autores coinciden en afirmar que se trata de un impuesto de tonalidades regresivas, ya que grava a todos los ciudadanos por igual, sin tener en cuenta su nivel de ingresos; además, el IVA se ha utilizado históricamente para compensar la pérdida de entrada de divisas derivada de la liberalización comercial que su-

\footnotetext{
${ }^{57}$ Ikhwanweb, "Freedom and Justice Party Views Regarding Egypt's IMF Loan", El Cairo, 27 de agosto de 2012.

${ }^{58}$ Idem.

${ }^{59} \mathrm{Idem}$.

${ }^{60}$ Hanieh, Lineages of Revolt, op. cit.
} 
pone la instauración de políticas neoliberales, con lo cual contribuye a la desindustrialización. ${ }^{61}$

A pesar de que el gobierno de los Hermanos Musulmanes presentó al FMI un programa de reformas que, en muchos sentidos, consideraba las condiciones impuestas por el organismo financiero internacional, el préstamo no llegó a concretarse. En julio de 2013, a un año de haber asumido el poder, el presidente Morsi fue depuesto por un golpe de Estado que restauró el gobierno militar en el país árabe.

El derrocamiento del presidente Morsi fue bien recibido por los mercados, y la bolsa cairota registró un alza de 7\%, "el mayor porcentaje ganado en un día en más de un año". ${ }^{62}$ Según analistas que comparten la perspectiva de que los Hermanos Musulmanes no poseían un programa económico de gobierno, el optimismo se debía a la certeza de que "los tecnócratas sabrían cómo lidiar con las instituciones, y ayudarían al país financieramente porque tienen una agenda clara". ${ }^{63}$

En dicho contexto auguraron la posibilidad de llegar a un acuerdo con el FMI. El organismo financiero leyó en la caída del gobierno del PLJ un paso más hacia la transición política, comenzada en febrero de 2011, e insistió en la necesidad de revisar el sistema de subsidios a la energía; los recortes a los mismos tuvieron lugar a mediados de 2014. En su informe, Arab Countries in Transition: Economic Outlook and Key Challenges, de octubre de dicho año, el FMI afirmaba que las proyecciones para la economía egipcia habían mejorado significativamente debido a la estabilización de la situación de seguridad y de la política nacional, y por la efectividad de los recortes. A partir de entonces, la presión del FMI sobre el nuevo gobierno fue en el sentido de profundizar las "reformas estructurales" a través del recurso al financiamiento externo.

Así, se felicitó al gobierno militar por haber tenido la valentía de tomar medidas que incluyeran el incremento de los

${ }^{61}$ Véase, por ejemplo, James Mahon, "Causes of Tax Reform in Latin America, 1977-1995”, Latin America Research Revierw, vol. 39, núm. 1, 2004, pp. 3-30.

${ }^{62}$ Matthew Davies, "Egypt Analysts Optimistic for Post-Morsi Economy", $B B C$ Neres, 5 de julio de 2013.

${ }^{63}$ Idem. 
precios de la energía por "entre 30\% y 150\%", ${ }^{64}$ a lo que se sumó el anuncio de la eliminación de la mayor parte de los subsidios en los siguientes cinco años. Asimismo, se subrayó la decisión del nuevo gobierno de realizar reformas impositivas que incluían la creación de un impuesto al valor agregado.

En febrero de 2015, el Fondo valoró, a través de su jefe de Misión en Egipto, Christopher Jarvis, que en los últimos meses el país norafricano había comenzado el camino para salir de la crisis. Uno de los grandes pasos en esta dirección había sido la reducción de los gastos fiscales. En este ámbito, los recortes a los subsidios fueron fundamentales, pero también resultaba de vital importancia el autocontrol que había comenzado a ejercer el Estado sobre los salarios del sector público, "los cuales, como resultado de una muy complicada estructura salarial, habían aumentado muy velozmente". ${ }^{65}$ Por otra parte, se subrayaba el hecho de que, por el lado de los ingresos, el programa fiscal presentado por el gobierno egipcio incluía la creación de un IVA; asimismo, se incentivaba la devaluación de la libra egipcia, a fin de hacer una economía más competitiva. El corolario necesario de la aplicación de estas políticas reformistas, se decía, sería aumentar el atractivo de Egipto para los capitales extranjeros.

\section{Conclusión}

Si bien no puede hacerse una afirmación taxativa y general que vincule a los movimientos islámicos con el neoliberalismo, tampoco puede afirmarse lo contrario, pues se trata de movimientos políticos marcados por los contextos en los que se despliegan. Por esta razón, es necesario que el investigador recurra a casos concretos, y a los discursos que envolvieron a estos movimientos cuando ocuparon cargos de decisión en el poder político. La coyuntura política, económica y social en la que llegaron al poder es, en este sentido, de fundamental impor-

\footnotetext{
${ }^{64}$ Fondo Monetario Internacional, Arab Countries in Transition: Economic Outlook and Key Challenges, Washington, 2014.

${ }^{65}$ Fondo Monetario Internacional, "Egypt: Steadfast Reforms Key for Economic Stability, Jobs, Growth”, IMF Survey Magazine: Countries E Regions, 11 de febrero de 2015.
} 
tancia. Al seguir este consejo, aquí se buscó reflexionar acerca de la práctica de gobierno de los Hermanos Musulmanes egipcios durante el año en el que estuvieron en el poder.

La lectura que primó en los medios de comunicación y los análisis occidentales respecto de la que fuera denominada Primavera Árabe, marco en el cual los Hermanos Musulmanes asumieron el poder en Egipto, no previó, o lo hizo en contadas ocasiones, el factor económico. Existió cierto consenso en el relato de un "despertar" brusco del pueblo árabe; como si de pronto, en un pase mágico, éste hubiera podido escapar de su "letargo" y se hubiera "dado cuenta" de que, al igual que otras naciones del mundo, también podía y quería vivir en democracia. Esta carencia explicativa permitió que los poderes extranjeros intentaran aplicar las mismas recetas que habían conducido a la crisis, esta vez en el marco de procesos democráticos. Los partidos políticos islámicos resultaron ser los vencedores de estos últimos, a pesar de la supuesta falta, que subrayaron las ya mencionadas lecturas hegemónicas de participación del factor religioso en los levantamientos.

Así, en el caso de Egipto, los Hermanos Musulmanes, bajo el rótulo del PLJ, lograron ocupar los máximos puestos de decisión política. En ese escenario, actuaron con precaución, pues buscaban, por un lado, no ahuyentar a las potencias liberales y a las instituciones internacionales y, por otro, evitar correr la misma suerte que quien había sido derrocado luego de 30 años en el poder, en el marco de una situación social, económica y política extremadamente delicada. Frente a quienes sostenían que no tenían un programa de política económica, o a quienes les endilgaban una inclinación hacia el modelo neoliberal, los Hermanos Musulmanes egipcios se aferraron fuertemente a ciertos principios de justicia social y redistribución de la riqueza que pueden ser rastreados en el corpus islámico y que constituyen la denominada economía moral.

Como se describió, el movimiento estudiado recurrió a los organismos de crédito internacionales constitutivos del neoliberalismo, pero no lo hizo porque creyera que fueran a solucionar los problemas económicos y sociales egipcios, sino porque los concibió como el único modo de generar confianza en los capitales internacionales y regionales, cuyas inversiones se hacían 
necesarias. No obstante, el PLJ, cuya plataforma electoral demostraba preocupación por el mejoramiento de los índices sociales a través de la distribución del ingreso, no pudo aceptar todas las condiciones de profundización del neoliberalismo que le imponía el FMI. La crisis económica, política y social que había heredado se intensificó debido a que la mano invisible del mercado, en alianza con poderes políticos regionales e internacionales preocupados por un gobierno de los Hermanos $\mathrm{Mu}$ sulmanes exitoso, cerró su puño sobre él.

La crisis comenzó a apaciguarse una vez derrocado el presidente Morsi y declarado terrorista el movimiento que lo había llevado a la presidencia. Entonces los capitales, sobre todo los de algunos países del Golfo - particularmente, Arabia Saudita, Emiratos Ârabes Unidos y Kuwait-, comenzaron a llover. Y una vez amainada la tormenta, las reformas estructurales que, de otro modo, habrían afectado aún más a la población, ensanchado la brecha de ingresos y alentado nuevos levantamientos, pudieron ser llevadas a cabo. En este caso, entonces, los Hermanos Musulmanes intentaron funcionar como una barrera a la aplicación de reformas encaminadas a profundizar el neoliberalismo egipcio, cuya crisis puede ser planteada como una de las condiciones de los levantamientos en primer lugar. En este sentido también se alejaron de la democracia liberal, tal como fue definida.

El rol de los países del Golfo fue también fundamental; países que han desarrollado un fuerte capitalismo financiero gracias a sus ingentes ingresos derivados de la exportación de productos energéticos. El excedente de los países de la península arábiga ha encontrado un excelente espacio de inversión en la economía regional. En el caso de Egipto, el sector bancario, el de agronegocios y el inmobiliario han sido puntos nodales de la neoliberalización de la economía, todos sectores en los que el capital del Golfo tiene una muy significativa posición. De esta manera, la débil barrera fue derribada por una conjunción de intereses políticos y económicos vinculados a la política neoliberal. Estos intereses, corporeizados en organismos internacionales como el Fondo Monetario Internacional y el Grupo de los Ocho y, asimismo, en las potencias liberales y las monarquías del Golfo, azuzaron al fantasma islámico para 
derrocar a los Hermanos Musulmanes y restaurar el gobierno neoliberal.

Dirección institucional de la autora:

Instituto de Relaciones Internacionales

Facultad de Ciencias Jurídicas y Sociales

Universidad Nacional de La Plata

48 e/ 6 y 7 - $5^{\circ}$ Piso - (1900)

La Plata, Argentina

\marielacuadro@yahoo.com.ar

\section{Bibliografía}

Azzam, Maha, "Egypt's Democratic Experiment: Challenges to a Positive Trajectory", Insight Turkey, vol. 15, núm. 2, 2013, pp. 157169.

CuADro, Mariela, "Historia del presente: las revueltas árabes a través del prisma de las Revoluciones europeas de 1989-1990 y de la Revolución Islámica de Irán”, Revista de Relaciones Internacionales, vol. 20, núm. 40, 2011, pp. 131-159.

Davies, Matthew, "Egypt Analysts Optimistic for Post-Morsi Economy”, $B B C$ Nerws, 5 de julio de 2013. [bbc.co.uk/news/business23183838, consultado en mayo de 2015.]

EL-AsHKer, Ahmed y Rodney Wilson, Islamic Economics: A Short History, Boston, Brill, 2006.

Fondo Monetario Internacional, Arab Countries in Transition: Economic Outlook and Key Challenges, Washington, 2014. [www.imf. org/external/np/pp/eng/2014/100914.pdf, consultado en mayo de 2015.]

Fondo Monetario Internacional, "Egypt: Steadfast Reforms Key for Economic Stability, Jobs, Growth", IMF Surven Magazine: Countries $\mathcal{E}$ Regions, 11 de febrero de 2015. [imf.org/external/pubs/ $\mathrm{ft} /$ survey/so/2015/CAR021115A.htm, consultado en mayo de 2015.]

Foucault, Michel, Nacimiento de la biopolítica, Buenos Aires, Fondo de Cultura Económica, 2007.

G8, "Declaration of the G8 on the Arab Spring", Cumbre de Deauville, Deauville, 26 y 27 de mayo de 2011. [mofa.go.jp/policy/ economy/summit/2011/declaration_arab.html, consultado en mayo de 2015.] 
HANIEH, Adam, Lineages of Revolt. Issues of Contemporary Capitalism in the Middle East, Chicago, Haymarket, 2013.

Hashemi, Nader, Islam, Secularism and Liberal Democracy. Toward a Democratic Theory for Muslim Societies, Nueva York, Oxford University Press, 2009.

Huntington, Samuel P., El choque de civilizaciones y la reconfiguración del orden mundial, Buenos Aires, Paidós, 1997.

Ikbwanweb, "FJP 2011 Program on Social justice”, El Cairo, 4 de diciembre de 2011. [fjponline.com/article.php? $\mathrm{id}=192$, consultado en junio de 2015.]

Ikbreanweb, “FJP's 2011 Program: Security, Economy, and Corruption as Urgent Issues”, El Cairo, 4 de diciembre de 2011. [fjponline. com/article.php? $\mathrm{id}=198$, consultado en junio de 2015.]

Ikbrwanweb, "Freedom and Justice Party Views Regarding Egypt's IMF Loan", El Cairo, 27 de agosto de 2012. [ikhwanweb.com/ article.php? id $=30263$, consultado en mayo de 2015.]

Ikbrwanweb, "Khattab: Muslim Brotherhood Economic Program is based on Free Market, Social Justice", El Cairo, 23 de agosto de 2012. [ikhwanweb.com/article.php?id =30258\&ref = search.php, consultado en junio de 2015.]

LEwIs, Bernard, La crisis del islam. Guerra Santa y terrorismo, Barcelona, Ediciones B, 2003.

Lipton, David, "Enabling Economic Transformation in the Middle East and North Africa", Londres, discurso en la London School of Economics, 13 de noviembre de 2012. [imf.org/ external/np/speeches/2012/111312.htm, consultado en mayo de 2015.]

MaHON, James, “Causes of Tax Reform in Latin America, 1977-1995”, Latin America Research Review, vol. 39, núm. 1, 2004, pp. 3-30.

Mouffe, Chantal, "Carl Schmitt y la paradoja de la democracia liberal”, Tópicos, núm. 10, 2002, pp. 5-25.

Moya Mena, Sergio, "El islam y sus manifestaciones sociopolíticas contemporáneas: breve introducción" (inédito).

Nagarajan, Karatholuvu, "Egypt's Political Economy and the Downfall of the Mubarak Regime", International Journal of Humanities and Social Science, vol. 3, núm. 10, 2013, pp. 22-39.

OrEn, Ido, “The Subjectivity of the 'Democratic' Peace: Changing U.S. Perceptions of Imperial Germany”, International Security, vol. 20, núm. 2, 1995, pp. 147-184.

OzAral, Basak, "Islam and Moral Economy”, en Tugrul Keskin (ed.), The Sociology of Islam. Secularism, Economy and Politics, Londres, Ithaca Press, 2011, pp. 21-44. 
Roy, Olivier, The Failure of Political Islam, Cambridge, Harvard University Press, 1994.

Roy, Olivier, "The Transformation of the Arab World", Journal of Democracy, vol. 23, núm. 3, 2012, pp. 5-18.

SADIKI, Larbi, Rethinking Arab Democratization: Elections without Democracy, Nueva York, Oxford University Press, 2009.

SAID, Edward, Orientalismo, Madrid, Libertarias, 1990.

SÁnchez GonZÁlez, Alberto y Carlos Calderón Modrego, "Banca islámica: un modelo de financiamiento alternativo para el desarrollo de África”, Relaciones Internacionales, núm. 26, junio-septiembre de 2014, pp. 69-84.

Sivan, Emmanuel, El islam radical. Teología medieval, política moderna, Barcelona, Bellaterra, 1997.

The White House, "Remarks by the President on the Middle East and North Africa", Washington, 19 de mayo de 2011. [whitehouse. gov/the-press-office/2011/05/19/remarks-president-middle-eastand-north-africa, consultado en marzo de 2016.]

Thomas, Scott, The Global Resurgence of Religion and the Transformation of International Relations. The Struggle for the Soul of the Treenty-first Century, Nueva York, Palgrave Macmillan, 2005.

TRIPP, Charles, Islam and the Moral Economy. The Challenge of Capitalism, Cambridge, Cambridge University Press, 2006.

VIDINO, Lorenzo, The Muslim Brotherhood after the Arab Spring: Tactics, Challenges and Future Scenarios, Bruselas, Wilfried Martens Centre for European Studies (Policy Brief), 2013. [martenscentre. $\mathrm{eu} /$ sites/default/files/publication-files/muslim_brotherhood web.pdf, consultado en mayo de 2015.]

Wallerstein, Immanuel, World-Systems Analysis. An Introduction, Durham-Londres, Duke University Press, 2004. 
